\title{
A Review of the Evidence for Intrinsic Ethnic Differences as Important Determinants of Skin Aging and Carcinogenesis: A Hope For All Ethnicities
}

Stephanie Chan, BS ${ }^{1}$, Jodie Sasaki, MD², Abrar Qureshi, MD, MPH ${ }^{1}$, Grace Giordano ${ }^{3}$, John $\mathrm{Koo}, \mathrm{MD}^{4}$, Nicholas Brownstone, MD ${ }^{5}$

${ }^{1}$ Department of Dermatology, Brown Warren Alpert Medical School, Providence, Rhode Island

2 Department of Dermatology, University of Washington Valley Medical Center, Seattle, Washington

${ }^{3}$ Barnard College, New York, NY

${ }^{4}$ Department of Dermatology, University of California San Francisco, San Francisco, California

${ }^{5}$ National Society for Cutaneous Medicine, New York, NY

\section{ABSTRACT}

By the year 2030, an estimated 1 in every 5 US residents will be over 65 years of age. Skin aging, which encompasses an entire spectrum of changes starting with fine rhytids (wrinkles), discolorations, and benign keratoses, progressing to deeper wrinkles, pre-cancerous lesions, and eventually skin cancers is of particular interest in dermatology. Skin pigmentation, or lack of it, is thought to be the most important determinant of how quickly (or slowly) people age. However, there is data suggesting that this may not be the case and other factors, such as ethnic differences in DNA repair may also play a major role. This article reviews available data on the differential aging process by ethnic background. The findings strongly suggest that there are other important determinants of aging besides skin color where future research may suggest ways to slow or reverse skin aging which can possibly benefit all ethnicities.

\section{INTRODUCTION}

Aging is an important and current topic in dermatology. By the year 2030 , over $20 \%$ of U.S. residents are projected to be 65 years or older, compared with $13 \%$ in 2010.[1] Skin aging encompasses an entire spectrum of changes starting with fine rhytids, discolorations, and benign keratosis, progressing to deeper rhytids, precancerous lesions, and eventually skin cancers. Ethnic differences in the aging process, including age of onset and severity of skin aging have been described. Defining the structural, functional, and biochemical differences in the skin of individuals from different ethnic backgrounds has been of interest in many fields including dermatology. Studies of pigmentary differences (size, distribution of melanosomes) have yielded the most consistent results, however studies regarding differences in other skin components have yielded inconclusive results.[2]

Skin pigmentation, or lack there of it, is thought to be the main determinant of how quickly (or slowly) people age. Many people, including dermatologists have traditionally assumed that the most important driver of 
skin aging is Fitzpatrick skin type (FST) and/or skin color. However, more recent data suggests that other factors such as ethnic differences in DNA repair may also play a key role in aging. Although darker skin types are less prone to the damaging effects of UV exposure, other factors may also influence the progression of skin aging. Here we present data suggesting that population differences in other genetic factors, such as ethnic differences in DNA repair and gene polymorphisms may be just as important as pigmentation in determining the propensity and pace of skin aging.

\section{METHODS}

Studies were identified by searching Pubmed and MEDLINE databases. Search terms included "skin carcinogenesis", "skin aging", "ethnicity", "DNA repair." We only included published articles in peer-reviewed journals that were written or translated in English. No year limits were applied and therefore included the full year range of the database until November 2020.

\section{RESULTS}

\section{Ethnic Differences in Skin Aging and Carcinogenesis}

It is well known that various ethnicities age differently. More specifically, several publications present data which suggest that the aging process in Asians begins significantly later than in Caucasians. In a cross-sectional study comparing the age of occurrence and severity of signs of skin aging of 256 women in Sendai, Japan and 280 women in Paris, France, rhytids occurred at comparable severity and frequency on average 15 years earlier in French women below 65 years old.[3] More specifically, rhytids, including expression lines on the forehead, frown lines, crow's feet, rhytids under the eyes and rhytids on upper lip were more pronounced at an earlier age in French women than in Japanese women. However, after 65 years of age the differences between the 2 populations are no longer significant. Comparing Chinese women again to French women a 10-year delay in rhytid onset in Chinese women was documented, supporting the ethnic population-related difference in the kinetics of rhytid severity.[4] Rhytid severity in French women followed a linear increase during aging while rhytid severity in Chinese women appeared to go through two stages - a slow increase until the age of 50 followed by a faster increase thereafter. In fact, after 60 years of age, the severity of rhytids was similar between both the Chinese and French population-in the same way that Japanese women matched their French counterparts after 65 years of age. Lastly, another study documented a similar result comparing wrinkle formation and skin sagging between Caucasians in America and Japanese in Japan.[5] As demonstrated in the above two studies, they also found that Japanese wrinkle scores were significantly lower than Caucasians in all areas of the face in younger age groups (20-49). However, wrinkle scores are similar by the age 50-60 years of age. Moreover, with regard to the most severe end of the skin-aging spectrum, skin cancer formation; there is also a large difference in risk of cancer by ethnicity. In a "head to head" comparison of nonmelanoma skin cancer among dermatology patients in Osaka University Medical Center as compared to patients from Colorado University, patients in Japan had $1 / 100$ of the risk of patients in the United States.[6] The rate of skin cancers in Caucasians compared to East Asians and Pacific Islanders (Japanese, 
Chinese, Korean, Hawaiian, Filipino) living in Hawaii was 45:1.[7]

\section{Individual Topology Angle (ITA)}

Individual Topology Angle (ITA) was developed as an objective classification of skin color based on colorimetric parameters $\mathrm{L}^{*}$ (luminance) and $\mathrm{b}^{*}$ (yellow/blue component). Although individuals with red hair and blue eyes have clearly lighter skin than Asian individuals, when a large population is studied, on average, according to the most rigorous objective quantification, there is significant overlap between Caucasian, Russian, Chinese and Japanese women (Figure 1).[8]

\section{Fitzpatrick Skin Phototype or Color Not Necessarily a Predictor of Skin Aging}

There are available data to suggest that the above difference in skin aging by ethnicity is not simply determined by the pigmentation of the skin; in fact, skin coloration may not even be the most important factor on skin aging. Here are the data documenting that, within non-Caucasian ethnic groups, some ethnicities with darker skin ages faster than lighter skin groups. Hillebrand et al. conducted a large population survey involving more than 2000 subjects in Los Angeles, Rome, London, Akita and Kagoshima (Japan). Surprisingly, with regard to signs of aging such as facial wrinkling, Asians showed the least amount of wrinkling, even as compared to African Americans.[9] As expected, Caucasians showed more wrinkling than either Asians or African Americans. Latinos showed wrinkling
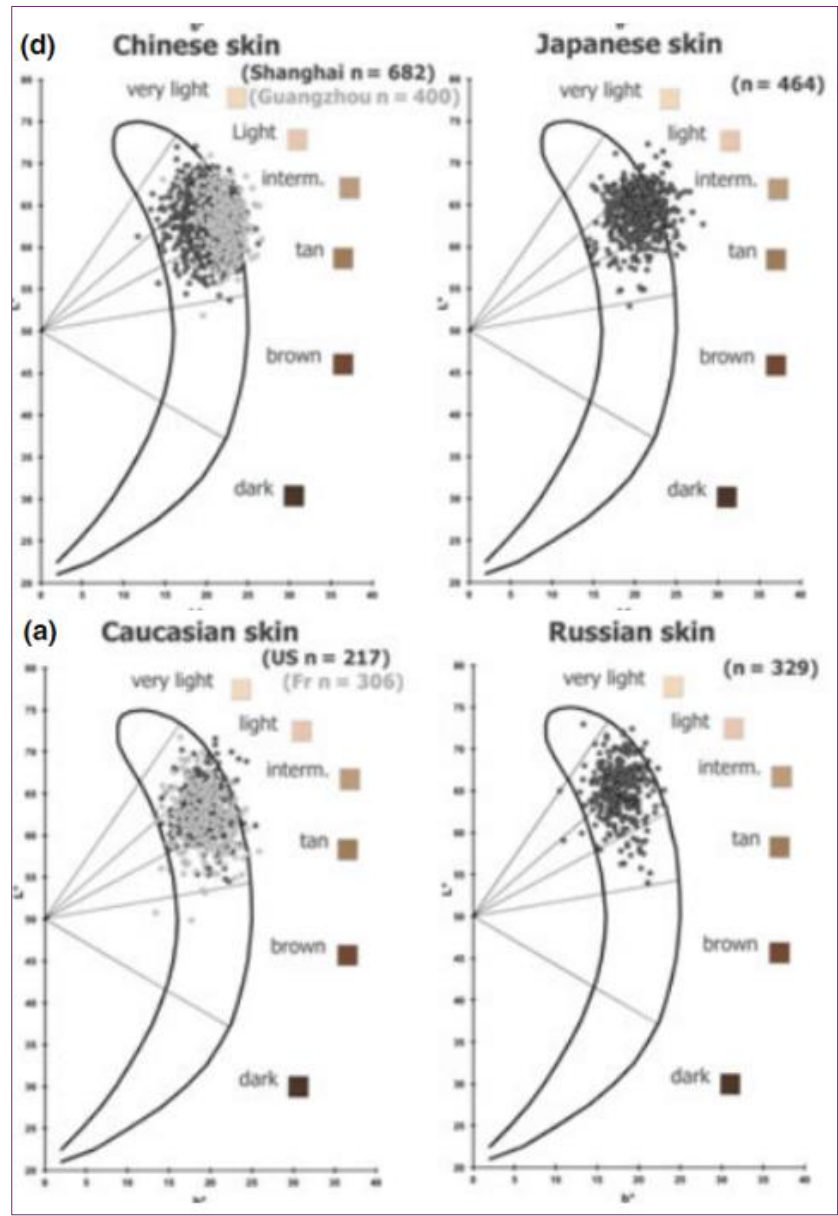

Figure 1. Individual typology angle (ITA) values of Caucasian, Russian, Chinese and Japanese Woman (Adapted from Del Bino S, Bernerd F. Variations in skin color and the biological consequences of ultraviolet radiation exposure. $\mathrm{Br} J$ Dermatol. 2013;169 Suppl 3:33-40. [PMID: 24098899].)

similar to African Americans, even though Latinos and Asians were similar in coloration as measured by the investigators. Interestingly, in this study, East Asians living in Los Angeles (Japanese, Korean, Chinese) showed no significant difference in facial wrinkling compared to women living in Japan. In another study, women in Bangkok, Thailand showed more severe level of wrinkling compared to Japanese or Chinese women despite the fact that Thai women were documented to be naturally more darkly pigmented than Japanese or Chinese women.[5,8] 


\section{DISCUSSION}

The reviewed evidence suggests that there are other intrinsic factors that may play a critical role in determining the aging process in addition to skin pigmentation. As documented above, there is data showing that certain ethnic groups with lighter skin, such as East Asians develop signs of aging such as facial wrinkling much later than African Americans. The data comparing the skin aging signs of Thai women when measured against Japanese and Chinese women also indicate that darker pigmentation does not always lead to less or slower skin aging. Moreover, the tremendous difference in skin cancer risk between Caucasian and Japanese as documented in "head-to-head" comparison between University of Colorado and University of Osaka cannot be fully explained by the difference in skin pigmentation when the skin pigmentation appears similar between these two groups when rigorously measured (ITA). It is traditionally believed that increased amounts of melanin better protect against UV-induced DNA damaged which then leads to decreased skin aging and carcinogenesis.[10] Sheehan Et. al. documented that UV-induced DNA damage occurred to the similar extent in individuals with FST II and FST IV skin; however, individuals with FST IV skin repair the experimentally induced damage more rapidly compared to those with FST II skin.[11]

What explains these notable differences between various ethnic groups regarding how their skin ages ranging from wrinkles to skin cancers? There are several possible explanations that underlie these differences. For one, Sheenhan Et. al. have documented difference in DNA repair capacity in skin by different FST. More specifically, after multiple exposures, the UV-induced DNA damage was repaired more rapidly among individuals with FST IV skin compared to FST II. The authors hypothesized that the more rapid repair may be due to a UVinducible sOS-like response previously described by several authors.[12]

There is also the possibility of genetically determined differences among ethnic groups in the robustness of the DNA repair mechanisms. Certain variants for melanocortin receptor (MC1R) are associated with an increased risk of NMSC independent of skin color, hair color, and self-reported phenotypic pigmentation.[13] Therefore, the differences in ethnic aging may be also be due to certain existence of the variants for MC1R.

Ethnic difference in telomere length and DNA repair capacity represents another possible area of research in anti-aging. In addition to the possible variation in DNA repair, it is well accepted that telomere shortening and damage is associated with the aging process. There is increasing evidence that telomerase, the enzyme that preserves the length of telomeres, may play a critical role in aging of the skin. The available data suggests that there are polymorphisms of telomerase[14]. Therefore, it is entirely possible that different ethnic groups (for example, Caucasians versus East Asians/Africans), may in fact have telomerase of varying efficacy that may explain the phenotypic differences in skin cancer risk and aging as documented above. Few preliminary studies have investigated telomere length and aging. Brown et. al. demonstrated that even though telomere length may differ amongst gender or race, it does not account for differences in

January 2022 Volume 6 Issue 1 


\section{SKIN}

population health outcomes[16]. Furthermore, a study on salivary telomere

Table 1. Non-topical agents that enhance DNA repair

\begin{tabular}{|c|c|c|}
\hline Non-Topical Agent & Mechanism of Action & Clinical Applications \\
\hline $\begin{array}{c}\text { Polypodium leucotomos } \\
\text { extract }\end{array}$ & $\begin{array}{l}\text { Decreases lipid } \\
\text { peroxides and } \\
\text { neutralizes } \\
\text { superoxide anions } \\
\text { and free radicals } \\
\text { Anti-inflammatory } \\
\text { properties - } \\
\text { Reduction of UV- } \\
\text { induced } \\
\text { cyclooxygenase-2, } \\
\text { p53 suppressor } \\
\text { genes, and } \\
\text { formation of } \\
\text { cyclobutene } \\
\text { pyrimidine dimers } \\
\text { (CPD) }\end{array}$ & $\begin{array}{l}\text { Prevention of polymorphous light } \\
\text { eruption and photodermatoses[15] } \\
\text { - Decreases acute pigment } \\
\text { darkening[16] }\end{array}$ \\
\hline Nicotinamide & $\begin{array}{l}\text { - Cofactor of ATP and } \\
\text { enhances DNA } \\
\text { repair }\end{array}$ & $\begin{array}{l}\text { - Treatment and prevention of } \\
\text { nonmelanoma skin cancers and } \\
\text { actinic keratosis[17] }\end{array}$ \\
\hline
\end{tabular}

Table 2. Topical agents that enhance DNA repair

\section{Topical}

Agents

\section{DNA Repair}

Enzymes

\section{Mechanism of Action}

\section{Photolyase}

- DNA repair enzyme splits cyclobutene; therefore reducing cyclobutene pyrimidine dimers (CPD) present[18]

- Shown to decrease Actinic Keratoses (AK)[19]

\section{Topical T4 endonuclease V}

- DNA repair enzyme derived from the UV-resistant microbe Micrococcus luteus

- Enhances DNA repair by removing cyclobutene pyrimidine dimers (CPDs)[20]

Green tea polyphenols

\section{Nicotinamide} (vitamin B3)
- Green tea has green tea polyphenols such as Epigallocatechin-3-gallate (EGCG)

- Induce DNA repair via induction of IL-12 keratinocytes

- Protects epidermal langerhans cells from UV damage and UV-induced damage[21,22]

- Essential coenzyme for cellular ATP production and DNA Repair

- Decreased nicotinamide slows DNA Repair[23,24] 
length did not account for race/ethnic differences in late life health and is not significantly associated with indicators of health among different races. These studies did not investigate telomere repair capacity specifically[17]. Therefore, the authors recommend future research to be focused on the ethnic differences in telomere and DNA repair capacity given the paucity of studies in this area. A review of the literature regarding skin aging and carcinogenesis revealed a significant difference among ethnic groups. Interestingly, the degree of skin pigmentation, even though important appears not entirely adequate to explain the documented differences in aging and the risk of carcinogenesis. A recent review on the lack of correlation between UV exposure and melanoma risk in skin of color also suggests that something other than UV exposure and skin pigmentation may be a more important determinant.[15]. A more advanced and accurate understanding of the skin aging process that goes beyond pigmentary differences may uncover new ways to minimize skin aging. Enhancing DNA repair through topical and oral treatments is already a reality through several of the following treatments. Oral agents such as polypodium leucotomos extract (PLE)[18] and nicotinamide (vitamin B3) and topical agents such as DNA repair enzymes and green tea polyphenols are all supplements that can enhance DNA repair (Table 1 and 2). These treatments may offer more hope to counter skin aging beyond skin pigmentation. Further research regarding differences in DNA repair mechanisms extends beyond the field of dermatology and examines how DNA repair mechanisms may affect neurological disorders. Recent research notes that patients with neurodegenerative disorders like Huntington's disease and Alzheimer's disease share an endophenotype of impaired DNA damage repair[19]. With the recent identification of this endophenotype researchers are now able to explore new opportunities in therapeutic interventions[20]. This demonstrates that this research and its potential therapeutic benefits are already being investigated for use in other disease states outside of cutaneous pathophysiology.

\section{CONCLUSION}

The differential aging process by ethnic background as well as basic science progress on skin aging was reviewed in this article. The data reviewed here indicates that skin aging is multifactorial in origin. While differences in skin aging and skin repair were highlighted here amongst different races, it is important to remember that we are all human beings. As such, the authors hope that the information presented here will educate readers and encourage future investigations on this topic with the goal of making use of the documented differential speed of aging between different ethnic groups as a "door of opportunity" that can lead us to find novel ways to mitigate skin aging and skin carcinogenesis to improve patient care and quality of life for people of all ethnicities.

Conflict of Interest Disclosures: None

Funding: None

Corresponding Author:

Stephanie Chan, BS

515 Spruce Street

San Francisco, CA 94118

858-776-1552

Email: stephanie_chan@brown.edu

References:

January 2022 Volume 6 Issue 1 
1. Office UCBPI. 2010 Census Shows 65 and Older Population Growing Faster Than Total U.S. Population - 2010 Census - Newsroom - U.S. Census Bureau. n.d.

https://www.census.gov/newsroom/releases/archi ves/2010_census/cb11-cn192.html (accessed 13 November 2020).

2. Del Bino S, Duval C, Bernerd F. Clinical and Biological Characterization of Skin Pigmentation Diversity and Its Consequences on UV Impact. Int J Mol Sci. 2018;19. [PMID: 30205563].

3. Tschachler E, Morizot F. Ethnic Differences in Skin Aging. In: Skin Aging. Gilchrest BA, Krutmann J, editors. Springer; 2006. p. 23-31.

4. Nouveau-Richard S, Yang Z, Mac-Mary S, et al. Skin ageing: a comparison between Chinese and European populations. A pilot study. J Dermatol Sci. 2005;40:187-93. [PMID: 16154324].

5. Tsukahara K, Fujimura T, Yoshida Y, et al. Comparison of age-related changes in wrinkling and sagging of the skin in Caucasian females and in Japanese females. J Cosmet Sci. 2004;55:351-71. [PMID: 15386027].

6. Tada M, Miki Y. Malignant Skin Tumors Among Dermatology Patients in University Hospitals of Japan. The Journal of Dermatology. 1984;11:313-21. [DOI: https://doi.org/10.1111/j.13468138.1984.tb01484.x].

7. Allison SD, Wong KL. Skin Cancer: Some Ethnic Differences. AMA Arch Derm. 1957;76:737-9. [DOI: 10.1001/archderm.1957.01550240055011].

8. Del Bino S, Bernerd F. Variations in skin colour and the biological consequences of ultraviolet radiation exposure. Br J Dermatol. 2013;169 Suppl 3:33-40. [PMID: 24098899].

9. HILLEBRAND $\mathrm{G}$. The age-dependent changes in skin condition in African Americans, Caucasians, East Asians, Indian Asians and Latinos. IFSCC Magazine. 2001;4:259-66.

10. Brenner M, Hearing VJ. The Protective Role of Melanin Against UV Damage in Human Skin. Photochem Photobiol. 2008;84:539-49. [PMID: 18435612].

11. Sheehan JM, Cragg N, Chadwick CA, Potten CS, Young AR. Repeated ultraviolet exposure affords the same protection against DNA photodamage and erythema in human skin types II and IV but is associated with faster DNA repair in skin type IV. J Invest Dermatol. 2002;118:825-9. [PMID: 11982760].

12. Gilchrest BA, Eller MS, Yaar M. Telomeremediated effects on melanogenesis and skin aging. J Investig Dermatol Symp Proc. 2009;14:25-31. [PMID: 19675549].
13. Han J, Kraft P, Colditz GA, Wong J, Hunter DJ. Melanocortin 1 receptor variants and skin cancer risk. Int J Cancer. 2006;119:1976-84. [PMID: 16721784].

14. Koziel JE, Fox MJ, Steding CE, Sprouse AA, Herbert B-S. Medical genetics and epigenetics of telomerase. J Cell Mol Med. 2011;15:457-67. [PMID: 21323862].

15. Lopes FCPS, Sleiman MG, Sebastian K, et al. UV Exposure and the Risk of Cutaneous Melanoma in Skin of Color: A Systematic Review. JAMA Dermatology. 2021;157:213-9. [DOI: 10.1001/jamadermatol.2020.4616].

16. Brown L, Needham B, Ailshire J. Telomere Length Among Older U.S. Adults: Differences by Race/Ethnicity, Gender, and Age. J Aging Health. 2017;29:1350-66. [PMID: 27469599].

17. Brown L, García C, Ailshire J. Does Salivary Telomere Length Explain Race/Ethnic Differences in Aging? Biodemography Soc Biol. 2019;65:351-69. [PMID: 33335644].

18. Nestor MS, Berman B, Swenson N. Safety and Efficacy of Oral Polypodium leucotomos Extract in Healthy Adult Subjects. J Clin Aesthet Dermatol. 2015;8:19-23. [PMID: 25741399].

19. Shiwaku H, Okazawa H. Impaired DNA damage repair as a common feature of neurodegenerative diseases and psychiatric disorders. Curr Mol Med. 2015;15:119-28. [PMID: 25732151].

20. Maiuri T, Suart CE, Hung CLK, et al. DNA Damage Repair in Huntington's Disease and Other Neurodegenerative Diseases. Neurotherapeutics. 2019;16:948-56. [PMID: 31364066]. 